\title{
Identifying, managing and supporting patients with a rare
}

\section{disease}

Rare diseases (RDs) are defined as diseases that affect fewer than 1 in 2000 people, $71.9 \%$ are genetic and $69.9 \%$ have symptom onset in childhood. There are an estimated 8000 rare diseases, and although individually rare, collectively they are common, with an estimated combined prevalence of 3.5$5.9 \%$, similar to diseases such as asthma and diabetes. Although rare diseases may be considered the domain of specialists, the identification, management and support of 'rare disease' patients and their families falls within the competence of GPs.

\section{Background}

Of the 8000 RDs, 100 diseases account for $80 \%$ of patients with RDs (see figure 1). RDs include familiar diseases, such as cystic fibrosis and haemophilia, and ultra-rare diseases, defined as affecting fewer than 1 in 50,000 , with often only a handful of identified patients worldwide.

\section{Figure 1}

Rare diseases are also referred to as orphan or neglected diseases reflecting the frequent paucity of information about the diseases, as well as a lack of treatments and research. This problem is globally acknowledged and although there is a long way to go, efforts to improve the outcomes for these patients are in place. Vocal and influential patient advocacy groups have led to change. The UK strategy for rare disease published in 2013, with more recent updates, clarifies the challenges and sets out a pathway to improve outcomes 'to ensure no one gets left behind just because they have a rare disease' (Department of Health and Social Care, 2020; Department of Health, 2013). Emerging technologies in both diagnostics and therapeutics have also improved the outcomes for many rare disease patients, with molecular diagnoses more accurately defining their problem and facilitating better 
treatments. Incentives for drug development and profitability, despite the small market, have led to an increasing number of approved drugs for RDs (EMA, E.M.A., 2020). Despite the breadth and number of rare diseases many issues and challenges are shared, allowing for a consistent approach.

\section{$\underline{\text { Identification }}$}

\section{When to suspect a rare disease?}

Frequently patients with RDs spend many years, or even decades, on their route to a diagnosis. This 'diagnostic odyssey', involves multiple referrals, investigations, and misdiagnoses, with many patients never receiving an accurate diagnosis. During this 'odyssey' patients receive inappropriate treatments, suffer from the consequences of disease progression and report the emotional distress of having unanswered questions and at times the legitimacy of their symptoms questioned. This is all at great burden and financial cost to the patient and the health care system (Eurordis, 2009). Indeed, frequent attendance in primary care can be considered a 'red flag' for an undiagnosed rare disease.

Even in the absence of a disease specific treatment, an accurate diagnosis is of immense benefit to the patient and their families. It enables the optimisation of their management, access to specialist care, the support of patient advocacy groups, access to clinical trials and emerging therapies, and importantly a diagnostic label and validation for their problems or experiences. Indeed, gaining access to health, educational and social support without accurate diagnosis (and something to put in the 'diagnostic box'), is incredibly difficult. Diagnosis can also herald the end of unbeneficial diagnostics, monitoring and treatment.

But how can a single clinician know about 8000 diseases? The short answer is, they can't. However, GPs can help. We offer whole patient care, are one of the few generalists working in the NHS and have continuity of clinical record over the life of the patients. We are familiar with excluding the rare amongst the common, excluding significant infections and cancers we may only see a 
handful of times in our career, and are well placed to see the broader clinical picture. We can join the dots spread both across the biology of the patient and the chronology of their record that may be missed by secondary care colleagues working in specialist 'siloes' (Evans \& Rafi, 2016). An inquisitive mind and the use of 'gut feeling' when patterns diverge from the norm should be a prompt for further enquiry (de Vries et al, 2018), with 'Family GENES' a useful mnemonic to frame such enquires (see Box 1).

\section{Box 1}

Certain scenarios are enriched with patients who may have an undiagnosed rare disease, including when multiple umbrella terms are used as diagnoses (e.g. learning disability, epilepsy, cerebral palsy) (Crowe et al, 2020). Ask yourself, does the umbrella term account for the full clinical picture? Or is the umbrella term capturing just one component of what is a much longer list of problems that the patient has?

Information about the patient's family history, can alert the GP to rare disease. GPs frequently are aware of the extended families registered at their practice, particularly in closely-knit communities. Collecting a family history has formed an integral part of general practice for decades (Qureshi et al. 2004; Zander et al. 1978), with this information typically collected at opportunities such as registration with the practice, prescribing contraceptives and during cardiovascular health checks. In genomics knowledge of a patient's health issues frequently impacts other family members. In the absence of the patient's explicit consent to share such information the clinician may find themselves in a difficult situation, balancing their duty to protect confidentiality, with the risk of causing harm to another family member. In primary care these scenarios are infrequent and if encountered, guidance should be taken, but a key message is that confidentiality is not absolute and breaches of confidentiality can be made in certain situations where significant risk may occur to another person (Middleton et al, 2020). Patterns of inheritance typically reflect the diseases that may appear in a comprehensive 
family history. Autosomal dominant conditions may be recognised by similar characteristics presenting in each generation, for example grandparents, uncles and cousins with abnormal limb movements may indicate Huntington's disease. Many rare diseases follow an autosomal recessive inheritance pattern and therefore may be difficult to identify from the family history. The exception is when parents and other couples in the close family are related to each other before marriage, for example cousins. We are all carriers for more than 20 of the approximately 1800 autosomal recessive disorders, however typically, pathogenic variants in the same gene are not inherited from both parents (Antonarakis, 2019). When there are consanguineous marital patterns, practised in several minority communities in the UK, there is an increased likelihood that the same pathogenic variants are inherited from both parents. Similar clinical features in several members of these families may indicate a rare recessive disorder e.g. childhood onset visual or hearing loss, learning disabilities or recurrent stillbirths. It may be perceived as challenging to take a detailed family history in these patients, due to cultural sensitivities and sometimes language barriers. Is this something you would feel able to do?

When clinical suspicions are heightened there are several resources available to help guide a problem-based search for differential diagnoses (see Box 2). These differential diagnoses can help guide subsequent investigation or referral, with advice often readily available through clinical genetics departments or, if an inherited metabolic disease is suspected, the local clinical biochemist or regional metabolic centre.

\section{Box 2}

\section{Clinical case scenario 1}

Salma is 22 years old and comes to see you with her mother and sister with an episode of acute sinusitis. Her family moved to the UK from Yemen 6 years ago. She has mild learning difficulties and well-controlled epilepsy; Mum attributes these problems to "being dropped on her head when she was a baby". You note from her records that in addition to lamotrigine, she is on long-term calcium and vitamin d supplementation following an episode 
of hypocalcaemia 4 years ago. You examine Salma and incidentally note that her facial features, although subtle, are quite different to her mother's and sister's. You advise that sinusitis is a self-limiting condition and symptomatic management is all that is required, and when to return to see you if it doesn't resolve or worsens. You make a note to yourself to explore her records more thoroughly at the end of the surgery, as her past history doesn't make sense to you.

On review of her notes you note she was seen once by neurology after arriving in the UK, the letter comments on the learning disability and a CT head was performed which was normal. You reflect on her combination of symptoms and her subtle facial dysmorphology and feel there may be a genetic syndrome to explain her problems. You input her clinical features into an online rare disease search engine and it gives 22q11.2 deletion syndrome (see figure 2), a chromosomal anomaly that can be identified by microarray, as a differential diagnosis. You feel this is plausible.

What would you do next? How would you approach and discuss this with Salma and her mother about your suspicions? How might you take into consideration cultural and religious background and beliefs into your discussions?

\section{Figure 2}

\section{$\underline{\text { Investigation and referral }}$}

Having identified features that suggest a rare disease a step-wise approach should be adopted to refine clinical suspicions and the appropriate next steps. This may involve initial investigations in primary care and referral into appropriate local pathways.

For example, tests that may be performed in primary care include:

- An elevated creatine kinase (CK) level for someone with myalgia in a range of inflammatory and metabolic muscular disorders.

- Abnormalities in electrolytes and a morning cortisol level for Addison's disease.

- Abnormality in the lipid profile for metabolic conditions such as familial hypercholesterolemia.

Few genomic tests can be directly requested in primary care. Referral offers the opportunity to clarify or confirm the diagnosis, with more focussed 
assessments and genomic testing available in clinical genetics and in specified clinical scenarios other specialties. Which genomic tests are commissioned by the NHS in England, when they should be performed and by whom has been standardised as part of the National Genomic Test Directory, and might include a chromosome analysis (microarray), single gene testing (e.g. in cystic fibrosis), and next generation sequencing (gene panels, whole exome or genome sequencing (WES/WGS).

GPs will be exposed to rare metabolic diseases through new-born screening (NBS). In the UK this screening involves testing for 9 rare diseases (see Box 3), measuring the metabolites or enzyme level in a dried blood spot from the heel prick test. Subsequent definitive testing is performed on abnormal results, in an often time critical manner. The adoption of diseases for inclusion is at the discretion of the UK National Screening Committee. Although the number of diseases has grown, there is significant international variability, with 40 diseases screened for in Italy and in the US 35 mandated, with some states testing for more than 50 diseases (Chan \& Petros, 2019).

\section{Box 3}

With significant advances in genomics and adoption of testing, an important consideration for primary care is when patients previously investigated, often as a child, and discharged to primary care for symptomatic management, should be referred back (or not) for a further attempt at establishing a diagnosis. For example, Microarray, also sometimes referred to as an array $\mathrm{CGH}$, is now widely used, frequently by paediatricians, as the first tier genetic test for patients with intellectual disability, congenital anomalies, dysmorphism and developmental delay. It can identify copy number variants (CNV), missing or duplicated segments of chromosomes, which are frequently the aetiology of these disorders (for a further explanation of microarray please refer to the Unique web page detailed in the resources (Box 4)). The national genetic testing directory advises which test should be performed in which clinical scenario and advises whole exome sequencing (WES) for unexplained congenital malformations, unexplained intellectual disability and global developmental delay often following a microarray and whole genome 
sequencing (WGS) for suspected ultra rare or atypical monogenic disorders. The additional diagnostic yield of these technologies for rare diseases can be substantial. In NHS England's 100,000 Genome Project, a cohort of rare disease patients who had been extensively investigated by multiple other methods prior to enrolment, WGS achieved a diagnosis in $26 \%$ of rare disease patients and for those with intellectual disability $37 \%$.

The decision making for whether to refer back or not is multifaceted involving the current level of disability, likelihood of benefit to the patient and the wishes of the patient and their family. Would you feel able to have such a discussion? Do you know where your local genetic departments are and if there are local referral protocols?

\section{Clinical case scenario 2}

Sarah is 28-years-old and comes to see you for preconception advice. She asks whether she should have a test for cystic fibrosis (CF) because her partner Dave is known to be a carrier and Dave's older brother died of CF when he was 17. (see figure 3) She wants to know what her risk of having a baby with CF is. What would you tell her?

She decides that she would like to be tested. What should you do next?

You review the National Genomic Test Directory and identify that one of the criteria for CF carrier testing is 'partner of a known CF carrier'. In your region there is a protocol to enable CF carrier testing in primary care for this indication.

You clarify with the laboratory what sample is needed, typically a blood test in an EDTA (purple) topped bottle, and that it takes approximately 2 weeks for a test result. You discuss with Sarah over the phone the implications of the test, that it identifies mutations responsible for approximately $90 \%$ of CF, but not all, so therefore doesn't completely exclude the chance of having a child with CF but does greatly reduce the risk. You advise if she is found to be a carrier, other family members may also be carriers and further guidance and testing could be arranged through the clinical genetics department. You gain her consent, record this in her notes and complete the form for her to collect and arrange the test.

Would you feel comfortable explaining autosomal recessive inheritance? Could you explain about the risk of having a child affected? Population 
carrier frequency for cystic fibrosis in those of North European heritage is approximately 1 in 25 .

\section{Figure 3}

If both parents are carriers of an autosomal recessive condition then each child has a 1 in 4 chance of inheriting both copies of the affected gene and therefore being affected, and a 3 in 4 chance of being unaffected ( 1 in 4 of inheriting two normal copies, and 1 in 2 chance of inheriting one normal and one affected copy). In this scenario we know Dave is a carrier, and assuming Sarah is of Northern European heritage she has a 1 in 25 chance of being a carrier. Therefore their risk of having a child affected by CF with no further testing is ( 1 in $4 \times 1$ in 25)=1 in 100. It is important to remember that pregnant couples with similar concerns should be offered an urgent referral for timely guidance and testing.

\section{Management and support}

Despite frequently experiencing a sense of relief once a diagnosis is received, this is often tempered/quickly replaced by frustration at a lack of accurate information, variability in the level and quality of care and often an absence of approved therapies or in some cases restricted access to treatment due to the cost. These frustrations with the care pathway are often shared with GPs.

\section{Emerging rare disease drugs}

RD treatments are frequently at the vanguard of new and emerging therapies and form a significant proportion of new drug approvals. For example, Ataluren for Duchenne muscular dystrophy is a small molecule that "reads through" the premature stop codon in the gene and makes a more normally functioning protein. Gene therapies have been approved for conditions such as a rare inherited retinopathy caused by mutations in the $R 65$ gene and for ADA-SCID, a rare form of immunodeficiency. The cost of some of these new 
therapies can be very high and may pose a reimbursement challenge to health services. An exciting area that helps address this is the repurposing of approved drugs for other indications, including for RDs.

Primary care can play a role in advising patients on clinical trial opportunities, directing patients to patient support groups and ensuring appropriate referral to specialist clinics, including nationally commissioned centres.

\section{Caring for rare disease patients in primary care}

For many patients, care may be complex involving multiple specialists often at distant regional or national specialist centres. Although specialist centres improve care and equity of access to emerging therapies and trials, this can be to the detriment of local disease knowledge and local health care system input for that patient. GPs can help and have a key role in the co-ordination of care and management of medically complex patients (RCGP, 2019). They can act as a local expert for that patient and their disease, navigating local health and social services. A range of resources to help support patients are included in Box 4. For rare disease patients, simple strategies can make a tremendous difference, some of these strategies are included in Box 5.

Box 4 and 5 here

\section{The future of rare disease in primary care}

There is an increasing ability to both diagnose and treat rare diseases. GPS currently play a crucial role and are likely to have an increasingly important role in this. The definition of a RD is likely to change. More RDs will be defined but common diseases will more often be broken into molecularly distinct subtypes. Will these subtypes be considered RDs?

\section{Disease identification and diagnosis}


Primary care records and GPs' skills set are especially well suited to playing a greater role in identifying patients with RDs. Enhanced data analytical methods are being used, including artificial intelligence (AI), to analyse primary care records for patterns of features that suggest a rare disease with suggested next steps for investigation (EAHSN, 2019). Primary care research databases can also be used to gain greater understanding of the natural history of rare diseases and their impact, as well as being used to develop predictive tools that intelligently target where resources should be placed to screen for such disease.

With greater use of direct to consumer testing (DTC), patients may present with genomic information reporting their carrier status for certain rare diseases. Although potentially valuable, these tests have limitations and frequently lack the sensitivity of carrier testing performed through NHS genetics laboratories, identifying the most common pathogenic variants only, and thereby potentially giving false reassurance. Would you feel comfortable explaining what this means to a patient and know when this may need further exploration? The joint position statement on DTC from the RCGP and BSGM may help inform how to approach this scenario.

www.rcgp.org.uk/policy/rcgp-policy-areas/genomic-position-statement.aspx

The number of diseases included in the NBS will expand, with WGS of all newborns likely to be the most economically prudent approach (van Campen et al, 2019). However there remain significant issues and uncertainties about the natural history of many diseases. Which parts of the WGS should be interrogated and for what diseases is less clear and as new genetic variation is identified, the significance these differing parts may be unclear and adds further uncertainty. Will GPs be equipped to communicate this complexity and uncertainty?

\section{Disease management and therapeutics}

RDs are already managed at nationally commissioned specialist centres, often some distance from the patient's home. Telemedicine and video 
consulting will play a greater role, with remote expert input into local care pathways. How will this fit into local health care and what role will there be for primary care? Will there be a primary care clinician, a GP with a specialist interest, to act as the co-ordinating doctor for a range of medically complex patients? This clinician could act as the key doctor for these patients incorporating the advice of national and local expertise with their generalist knowledge and awareness of local services.

A growing number of RDs have licensed therapies and clinical trials for emerging treatments. These drugs, often at the forefront of therapeutic advances and headline making news, are frequently prohibitively expensive. How will health care systems afford them and what level of priority should the NHS put on ensuring patients access to these therapies?

\section{$\underline{\text { Key points }}$}

- Rare diseases are individually rare but collectively common

- Primary care plays a key role in identifying patients who would benefit from referral to specialists and their management day-to-day, as well as communicating and advocating for these patients between different health and social care services

- "Dare to think rare" and be inquisitive; is there a more plausible explanation for your patient's problems?

- Use online resources and local experts and clinicians to refine your suspicions

- Consider patients for whom revisiting their diagnosis would be worthwhile given advances in disease knowledge and diagnostics

- Work collaboratively with rare disease patients, they are often experts in their disease and how it affects them

\section{References and further information}

(EMA), E. M. A. (2020) Orphan designation: an Overview, 2020. Available online: www.ema.europa.eu/en/human-regulatory/overview/orphandesignation-overview [Accessed 09 June 2020). 
Antonarakis, S. E. (2019) Carrier screening for recessive disorders. Nature Reviews Genetics, 20(9), 549-561. doi:10.1038/s41576-019-0134-2

Department of Health (2013) The UK Strategy for Rare Diseases. Available online:

https://assets.publishing.service.gov.uk/government/uploads/system/uploads/ attachment data/file/260562/UK Strategy for Rare Diseases.pdf [Accessed 09 June 2020)

Department of Health and Social Care (2020) The UK strategy for rare diseases: 2020 update to the implementation plan for England - dhsc-2020update-to-the-rare-diseases-implementation-plan-for-england.pdf. Crown copyright. Available online:

https://assets.publishing.service.gov.uk/government/uploads/system/uploads/ attachment data/file/867940/dhsc-2020-update-to-the-rare-diseasesimplementation-plan-for-england.pdf [Accessed 09 June 2020)

Chan, K. \& Petros, M. (2019) Simple Test, Complex System: Multifaceted Views of Newborn Screening Science, Technology, and Policy. Global Pediatric Health, 6, 2333794X19894812. doi: 10.1177/2333794X19894812

Crowe, A., McAneney, H., Morrison, P. J., Cupples, M. E. \& McKnight, A. J. (2020) A quick reference guide for rare disease: supporting rare disease management in general practice. British Journal of General Practice, 70(694), 260-261. doi: 10.3399/bjgp20X709853

de Vries, E., Fransen, L., van den Aker, M. \& Meijboom, B. R. (2018) Preventing gatekeeping delays in the diagnosis of rare diseases. British Journal of General Practice, 68(668), 145-146. doi: 10.3399/bjgp18X695225

EAHSN (2019) Mendelian secures £500k to help diagnose rare disease patients - Eastern AHSN, 2019. Available online:

www.eahsn.org/news/2019/02/mendelian-secures-500k-to-help-diagnoserare-disease-patients/ [Accessed 09 June 2020).

Eurordis (2009) The Voice of 12,000 patients. Experience and Expectations of Rare Disease Patients on Diagnosis and Care in Europe. Available at: www.eurordis.org/publication/voice-12000-patients (accessed 01 October 2020)

Evans, W. R. H. \& Rafi, I. (2016) Rare diseases in general practice: recognising the zebras among the horses. British Journal of General Practice, 66(652): 550-551. doi: 10.3399/bjgp16X687625

Middleton, A., Christine, P., Jonathan, R et al .(2020) Professional duties are now considered legal duties of care within genomic medicine. European Journal of Human Genetics, 28, 1301-1304. DOI: 10.1038/s41431-0200663-3 
RCGP. Clinical topic guide: Genomic medicine. Available at: www.rcgp.org.uk/training-exams/training/gp-curriculum-overview.aspx (accessed 09 June2020)

van Campen, J. C., Sollars, E. S. A., Thomas, R. C., et al. (2019) Next Generation Sequencing in Newborn Screening in the United Kingdom National Health Service. International Joournal of Neonatal Screening, 5(4): 40. doi: 10.3390/ijns5040040.

\section{Conflict of interest}

William Evans is the chairman of a rare disease charity NPUK and clinical lead at Mendelian. A company using technologies to improve rare disease diagnosis.

Lucy McKay is the CEO of a rare disease charity M4RD advocating for greater awareness of rare diseases amongst health care professionals.

Nadeem Qureshi is a member of the NICE guideline development groups for familial hypercholesterolaemia, familial breast cancer and lipid modification. Chief investigator on MRC and NIHR grants on familial hypercholesterolaemia and familial breast cancer. 
Figure 1. Rare diseases in numbers.
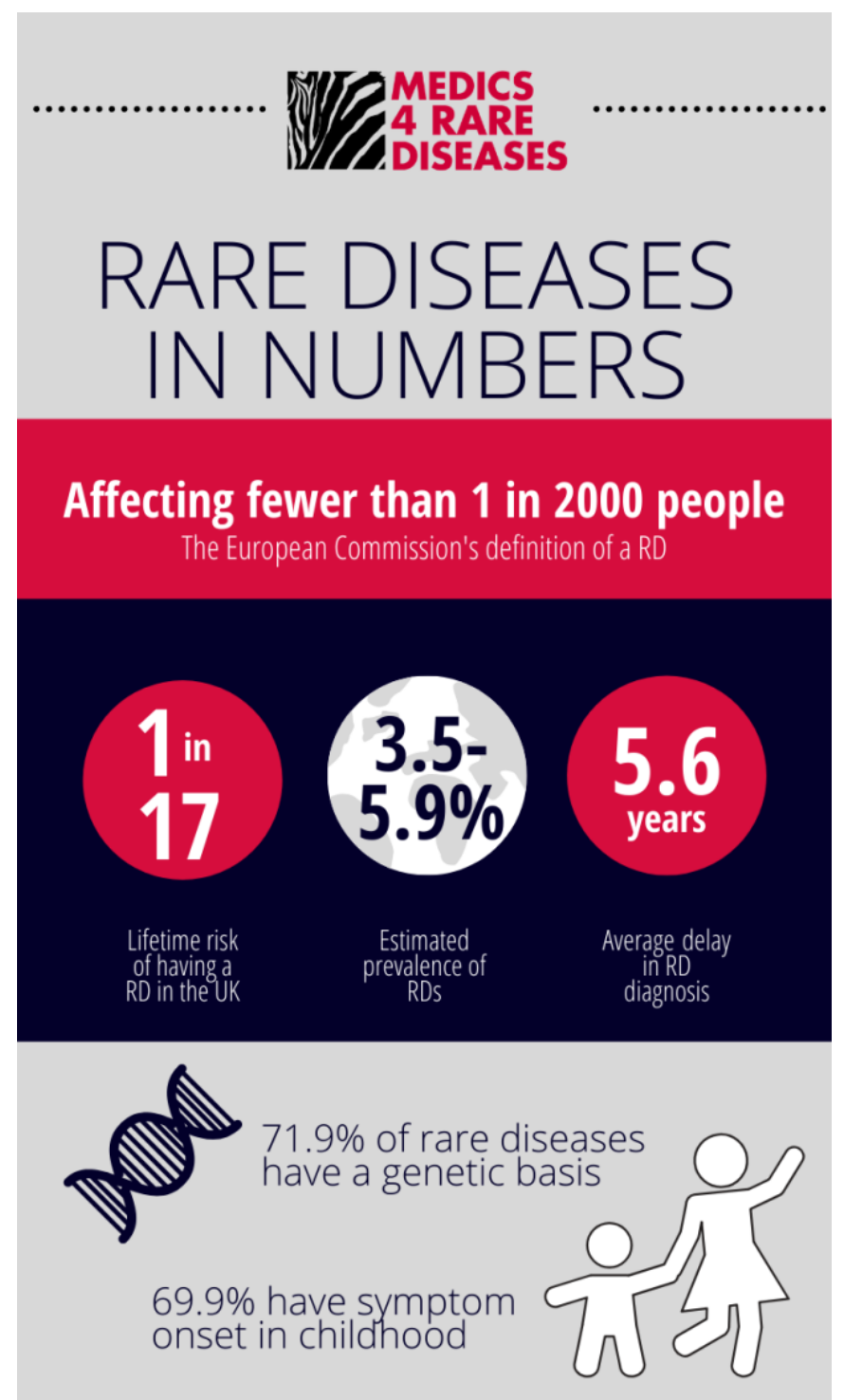

\section{ALL CHILDHOOD CANCERS ARE RARE DISEASES}

Zebra imagery has united the rare disease community across the world because of the old adage "hear hooves...think horse. Not zebra."

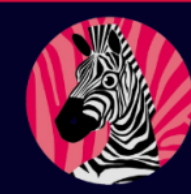

M4RD is equipping medical students \& doctors with the knowledge and tools they Visit m4rd.org need to \#DareToThinkRare $\quad \vdots$ videos \& opportunities 


\section{Box 1. FAMILY GENES}

Scenarios to suspect a rare or genetic disease in primary care

Family history: 3 generation FHx. Multiple affected siblings or individuals in multiple generations. (Absence of a FHx does NOT rule out genetic causes.)

- G: group of congenital anomalies. Common anatomic variations are common; but two or more anomalies are much more likely to indicate the presence of a syndrome.

- E: extreme or exceptional presentation of common conditions. Early onset CV disease, cancer, or renal failure. Unusually severe reaction to infectious or metabolic stress. Recurrent miscarriage.

- $\mathbf{N}$ : neurodevelopmental delay or degeneration. Developmental delay in the paediatric age group carries a very high risk for genetic disorders. Developmental regression in children or early onset dementia in adults should similarly raise suspicion for genetic etiologies.

- E: extreme or exceptional pathology.

- S: surprising laboratory values. Markedly abnormal pathology results.

Is there a more plausible explanation for my patient's problems?

(Whelan et al. 2004) 


\section{Box 2. Rare disease diagnostic resources}

- MendelApp is a rare disease semantic search engine. Type in 2 clinical features and it maps to a number of rare disease differential diagnoses. Further questions are asked to for further refinement. www.mendelian.co

- The Phenomizer. An online diagnostic engine where clinical terms using Human Phenotype Ontology (HPO) terms are combined to generate a differential diagnostic list. http://compbio.charite.de/phenomizer.

- Findzebra is a rare disease search engine that has been shown to outperform Google. www.findzebra.com.

- Orphanet a European rare disease resource with information about diagnosis, diseases, genetics and specialist centres. www.orpha.net 
Figure 2 Mendelian's Mendelapp search output

mendelian

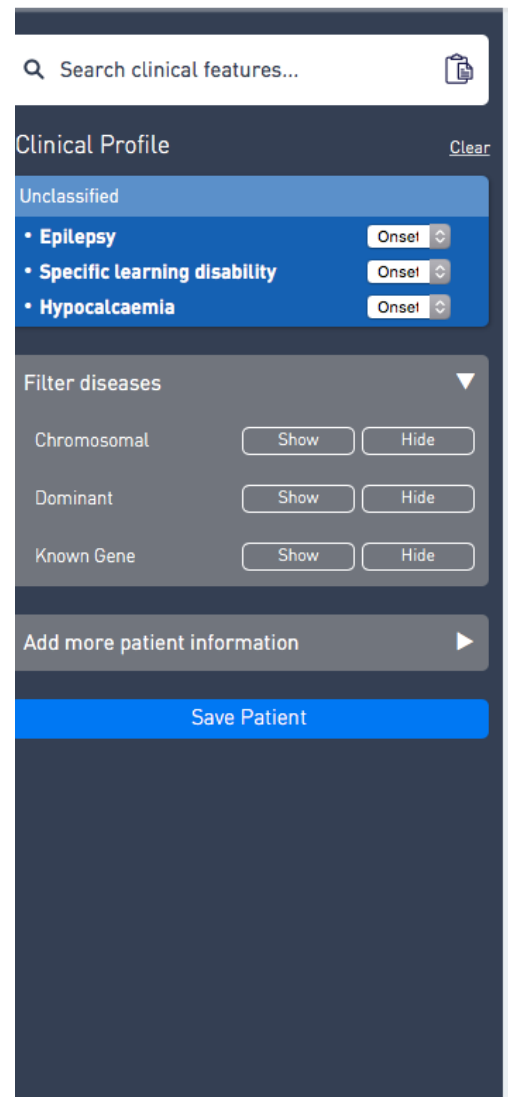

EN ES Update Consent

My Patients

Log Out

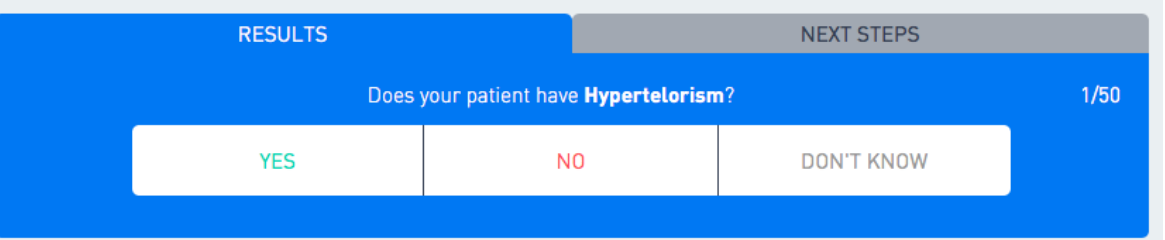

Share | Download $\nabla$

VELOCARDIOFACIAL SYNDROME DIGEORGE SYNDROME; DGS

\begin{tabular}{l|l} 
TBX1 & TBX1
\end{tabular}

Also known as: Shprintzen vcf syndromelvcfs|chromosome DiGeorge syndrome (DGS) comprises hypocalcemia arising 22q11.2 deletion syndromelvcf syndrome... from parathyroid hypoplasia, thymic hypoplasia, and outflow

Feedback

22Q11.2 DELETION SYNDROME

HIRA, GP1BB, JMJD1C, ARVCF, SEC24C, COMT, UFD1 RREB1, TBX1

22 q11.2 deletion syndrome (DS) is a chromosomal anomaly

which causes a congenital malformation disorder whose

common fea...

OSTEOPETROSIS-

HYPOGAMMAGLOBULINEMIA SYNDROME

TNFRSF11A

Osteopetrosis-hypogammaglobulinemia syndrome is an

extremely rare primary bone dysplasia with increased bone

2P21 MICRODELETION SYNDROME

CAMKMT, PREPL, SLC3A1, PPM1B

The $2 p 21$ microdeletion syndrome consists of cystinuria,

neonatal seizures, hypotonia, severe growthand

developmental del... tract...

PRIMARY HYPOMAGNESEMIA WITH

SECONDARY HYPOCALCEMIA

TRPM6

Primary hypomagnesemia with secondary hypocalcemia

(PHSH) is a form of familial primary hypomagnesemia (FPH,

see this te.

RING CHROMOSOME 10 SYNDROME

Ring chromosome 10 syndrome is characterized by

intellectual deficit, growth retardation, and various

dysmorphic feature...

Seen in 16 published cases

AUTOSOMAL RECESSIVE KENNY-CAFFEY SYNDROME

TBCE

A rare, primary bone dysplasia characterized by pr postnatal growth retardation, short stature, cortical

\section{Box 3. Newborn blood spot screening}

Taken when 5 days old (can be up to 8 days old). With test results returned within 6 weeks (but sooner if the result is positive).

9 conditions tested for:

- Sickle cell disease (SCD),

- Cystic fibrosis (CF),

- Congenital hypothyroidism (CHT)

and six inherited metabolic diseases (IMDs):

- Phenylketonuria (PKU),

- Medium-chain acyl-CoA dehydrogenase deficiency (MCADD),

- Maple syrup urine disease (MSUD),

- Isovaleric acidaemia (IVA),

- Glutaric aciduria type 1 (GA1)

- Homocystinuria (pyridoxine unresponsive) (HCU). 
Figure 3. Pedigree for Sarah and family.

1590704370987-Case 2
$5 / 28 / 20$
$\square$ Cystic Fibrosis $\square$ CF carrier

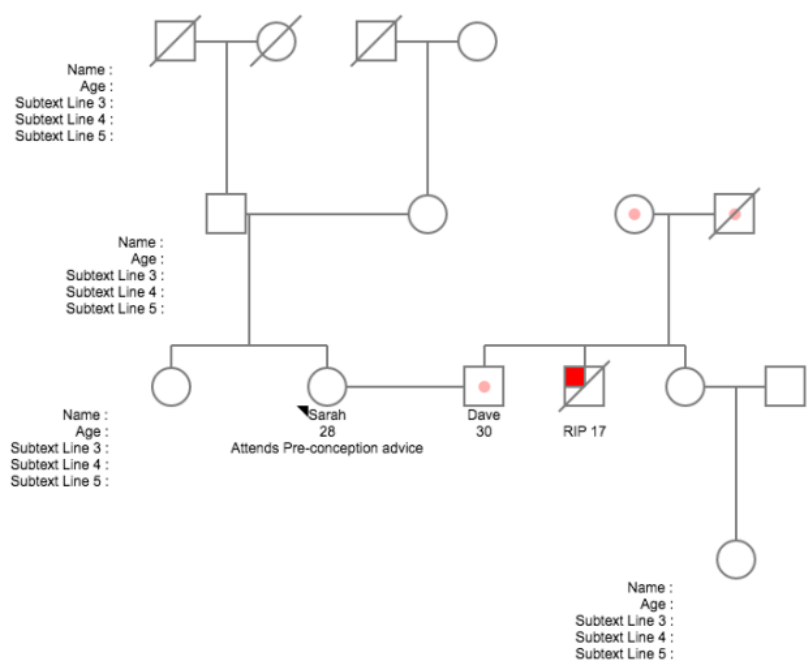

Box 4. Resources

- Orphanet a European resource of a range of resources for rare diseases. www.orpha.net/consor/cgi-bin/index.php

- Genetic Alliance brings together patient organisations for a range of rare diseases. They also run SWAN a support network for families with undiagnosed genetic conditions. https://geneticalliance.org.uk

- GeneReviews. Peer reviewed and updated published articles for a wide range of genetic conditions.

- Unique a charity that provides information for both families and health care professionals on chromosomal abnormalities. www.rarechromo.org

- Medics 4 Rare Diseases (M4RD) an organisation aimed at raising awareness and encouraging the engagement of healthcare professionals in rare diseases. www.m4rd.org/

- National Genomic Test directory www.england.nhs.uk/publication/national-genomic-test-directories/ 
- RCGP Genomic webinars (including a rare disease webinar). www.rcgp.org.uk/clinical-and-research/our-programmes/genomicswebinars.aspx

- Findacure a charity to support rare disease charities, encourage and support drug repurposing for rare diseases. www.findacure.org.uk

\section{Box 5 - Strategies for caring for patients with a rare disease in primary care}

- Patient summaries, emergency care plans, or useful recent letters highlighted or linked from the patient's EHR home page can ensure the patient feels you understand their disease and challenges. Patients and carers often report the emotional strain of having to explain their disease repeatedly to different health care professionals, and reliving a path to diagnosis that may have been traumatic and upsetting.

- Understanding and appreciation for each other's expertise. Patients and carers are frequently experts in the disease and how it affects them. As a GP, you are skilled in managing medical complexity, uncertainty and common diseases in your locality. A patient/doctor collaboration with shared decision-making in both diagnostic work up and management is both rewarding and optimises outcomes for the patient.

- Avoiding the easy assumption that every problem is a result of the 'headline' diagnosis. Rare disease patients have normal disease and infections too. For example unrecognised urinary retention, constipation or depression are common and treatable, with substantial improvements available in both the patient's quality of life and often their apparent disease severity

- Think holistically, often rare disease patients have complex multisystem disease. Don't assume that others have thought about and addressed simple things. Consider a patient from head to toe. 
Do they have dental issues that need addressing? Do they need speech and language therapy or dietician involvement? Do they need wheelchair services, physiotherapy or OT involvement? Do they have or need continence products, are these prescribed or are they purchasing themselves?

- Rare diseases are complex, significant and often life limiting diseases. Patients and their families may approach primary care for support with the multifaceted nature of these diseases. The care demands on can be substantial, with the need for both practical and psychological support for the family including their unaffected siblings. Signposting to patient advocacy groups (see box 4) can be invaluable. Early referral to local hospice services for life-limiting conditions, is encouraged. Children's hospices for example offer not only expert symptom management but also respite care, practical and psychological support to the whole family. 